\title{
Cureskin - Skin Disease Prediction using MobileNet Model
}

\author{
N. Divya*, Deepthi P Dsouza, Hariprasad \\ Department of Computer Science, Srinivas Institute of Technology, Mangalore, Karnataka, India
}

\begin{abstract}
Article Info

Volume 7, Issue 4

Page Number: 32-37

Publication Issue :

July-August-2021

\section{Article History}

Accepted : 01 July 2021

Published : 05 July 2021

Skin diseases are getting more common than any other disease in the world. Due to lack of personal care and different environmental factors many of the people are suffering from skin diseases. It may have caused because of infection, allergy, bacteria or viruses, etc. Not every patient has the facility to go to the doctor for primary consultation based on the financial issues. To overcome this problem we developed an android application which helps the patients in diagnosing the disease easily at home. There are several methods or algorithms in machine learning to make this process easier. We proposed an approach to skin disease prediction using MobileNet model which is a part of Convolutional Neural Networks (CNN). In total there are six diseases namely acne, actinic, psoriasis, tinea ringworm, eczema and seborrhoea. Our model is pre-trained by feeding thousands of images also including images which are not diseased and also which do not comes under skin. Our approach is simple, fast and inexpensive and does not require huge equipment for the diagnosis. It is found that MobileNet model gives best accuracy.

Keywords : Skin diseases, Machine Learning, MobileNet Model, Convolutional Neural Networks (CNN)
\end{abstract}

\section{INTRODUCTION}

As the human skin plays a huge role in their physical appearance and also counted as one of the largest part of the body. Hence, it is very important to keep our skin healthy and safe. In this paper, we will show how our proposed method works and how efficient the model is.

In India, skin diseases is gradually getting more and still people living in rural area are unaware of it. Especially, the causes or treatment are not known to them due to illiteracy, lack of personal hygiene, lack of awareness, pollution, etc. According to the research, around 1.9 billion people from all around the world are suffering from a skin disease at a particular time. Due to the shortage of dermatologists, many people consult general practitioners instead. As there are many skin diseases it will be very difficult for dermatologists to diagnose the disease. Many skin diseases does not have any symptoms during initial days, but could lead to a major problems like skin cancer and etc. In many cases even dermatologists fail to understand and predict the disease, so using computer technology is much important to help lessen the burden from the dermatologists. With the help of emerging technologies and computing we can

Copyright: @ the author(s), publisher and licensee Technoscience Academy. This is an open-access article distributed under the terms of the Creative Commons Attribution Non-Commercial License, which permits unrestricted non-commercial use, distribution, and reproduction in any medium, provided the original work is properly cited 
help the medical field. Machine Learning is becoming very popular in the field of medicine to help the doctors and reduce their work.

\section{LITERATURE SURVEY}

Jessica Vellasco, Cherry Pasicon, Jonathan Apuang propsed the method where the MobileNet model was used by smearing transfer learning on the 7 skin diseases to generate a skin disease classification system on Android application. The exponents assembled a total of 3,406 images and it is considered as unfair dataset because of the uneven number of images on its classes. Using dissimilar sampling method and pre-processing of input data was discovered to further improve the accuracy of the MobileNet. After using under-sampling method and the default pre-processing of input data achieved an $84.28 \%$ accuracy. While, using imbalanced dataset and default pre-processing of input data $93.6 \%$ of accuracy is achieved. The researchers explored oversampling the dataset and the model attained a $91.8 \%$ accuracy. In conclusion, by using oversampling technique and data augmentation on pre-processing the input data provide a $94.4 \%$ accuracy and this model was deployed on the developed Android application [1]

In this paper, Ms. Seema Kolkur, Dr. D.R.Kalbande, Dr.Vidya Khakar discussed dissimilar classifiers for skin disease detection. As the digitalization and improvement in technology is happening, more and more detailed data is becoming accessible. The techniques like Medical data mining can be used for diagnosis, and decision making, etc. The collection of original data related to 10 common skin diseases and applied five dissimilar classifiers for classification of an input record to one of these 10 diseases. Results specify these classifiers do well in identification of disease. This system can help new medical practitioners to correctly identify disease in case of doubt [2]
In the age of gadgets, machine learning is finding its application in all fields and clinical diagnosis is the latest calculation to it. Evolving ML based analytical tools are quite exciting to develop and it is authoritative to choose the right decision-making algorithm to achieve a better diagnostic accuracy. Hence, it is equally authoritative to select the right feature and machine learning algorithm to find the peak diagnostic precision. Image processing and machine learning based readings are being used in several areas such as face recognition, fingerprint recognition, discovery of tumor and separation. Here diverse $\mathrm{ML}$ algorithms are used for the classification tasks in these areas. The generally used $\mathrm{ML}$ algorithms are K-Nearest Neighbour (KNN) and kmeans algorithms. The choice of input feature is vigorous in any classification task, using ML algorithms. We discover that in previous studies, investigators have used diverse types of features, responsible on the precise classification tasks. In ordering images of skin diseases, different types of colour and texture features were used with $\mathrm{KNN}$ algorithms and obtained good exactness. [3]

Nawal Soliman ALolifi ALEnezi proposed an approach in which the system is implemented in MATLAB 2018b. We used Intel Core i3 processor with $2.10 \mathrm{GHz}$ having 4- GB RAM. At first, the input images are pre-processed, then features are extracted using pre-trained CNN. Finally, classification is performed using SVM classifier. Here, 100 skin images were used by numerous dermatological disease patients, also were taken from the Internet. The projected system can effectively detect 3 dissimilar skin diseases with an accuracy of 100\%. 20 images are used for validation purpose and 80 images for training purpose. The system works well. The recognition rate of our system is $100 \%$. The recognition rate of diseases is very high $100 \%$. [4]

In this paper, Shuchi Bhadula, Sachin Sharma, Piyush Juyal, Chitransh Kulshrestha showed how the use of 
five distinct machine learning classifiers could identify three kinds of skin diseases called acne, lichen planus and sjs ten. For further classifications, the skin image dataset is originally pre-processed. The dataset is split into the dataset of instruction and testing: 80 percent for instruction and 20 percent for testing. The testing data is trained into three different classes denoted as class a, b and c for three diseases. Afterwards, skin diseases are detected using five different classification algorithms called machine learning, logistic regression, kernel SVM, naive bayes, random forest, and CNN. Each algorithm runs on the same dataset ten times and the training accuracy is calculated for each run. Skin diseases are detected afterwards using five different classification algorithms called machine learning, logistic regression, SVM kernel, naive bayes, random forest, and CNN. Each algorithm runs ten times on the same dataset and each run calculates the training precision. CNN is a fully linked neural feed-forward network consisting of several layers of unidirectional linked nodes, often trained by back propagation. This multilayer architecture operates to extract, refine and classify prominent characteristics using various layers from a set of multidimensional input image datasets and provides more precise predictions. [5]

\section{Methodology}

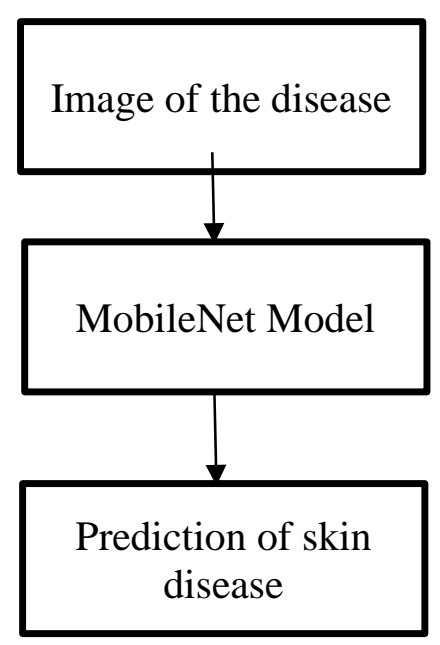

The focus of the project is to divide the images of skin diseases in six plus two different classes based on the type of diseases. Then the images are divided into train, test and valid for further classification. The images are trained using MobileNet model in the ratio of $80: 20$, where $80 \%$ of the images are used for training the model and $20 \%$ for testing the accuracy. The process initiates by accepting the user input in the form of image where the pre-trained MobileNet model will classify the disease. At last image of the disease and recognized disease name or, normal skin or not a skin will be displayed.

\section{A. Dataset Collection}

A total of thousand and twelve picture of diseases were possessed from online platform for each classes. There are totally 8 classes comprising more than thousand images each. Those eight classes include six diseases and one more of normal skin and the other of not skin.

Table 1: List of disease names

\begin{tabular}{|l|l|}
\hline Sr. No. & Disease Name \\
\hline 1 & Acne \\
\hline 2 & Actinic \\
\hline 3 & Eczema \\
\hline 4 & Psoriasis \\
\hline 5 & Seborrhoea \\
\hline 6 & Tinea ringworm \\
\hline
\end{tabular}

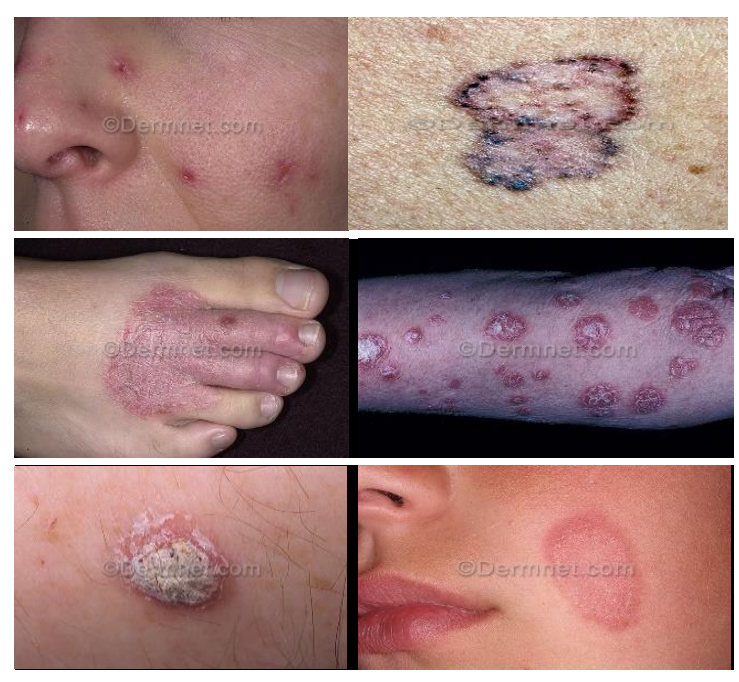



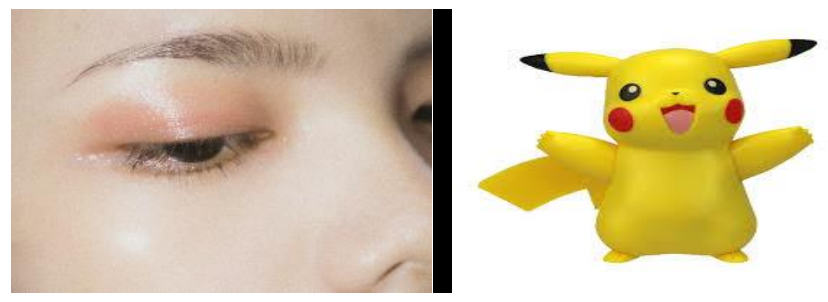

Fig: The images shows acne, actinic, eczema, psoriasis, seborrhoea, tinea ringworm, normal skin and not a skin respectively.

\section{B. Image Resizing}

The images collected from various platform will be in different dimensions. We must either increase or decrease the size of it. The dimensions of picture obtained are different in terms of sizes but then rescale to $224 \times 224$ pixels. By performing image resizing it reduces the time taken for processing it and hence the system gives better performance.

\section{Framework}

Tensor flow open source and end-to-end platform. Used for numeric computation and has libraries, flexible ecosystem of tools. Construct and train model easily using high-level APIs. Keras is one library among multiple tensorflow libraries. Tensor flow has both high and low APIs wherein keras provide only high-level APIs. Keras built in python so it's much user-friendly than Tensor flow. Tensor Flow permit for training on both a CPU and GPU. Our model is implemented using CPU.

D. Pre-processing

Initially the images in the dataset is rescaled to $224 \times 224$ pixels then converting the image into an array. We will feed the images to the mobilenet model and receive the output in the form of an array. Output will be given in terms of percentage, the one with maximum value is considered as final output.
E. Flow Diagram

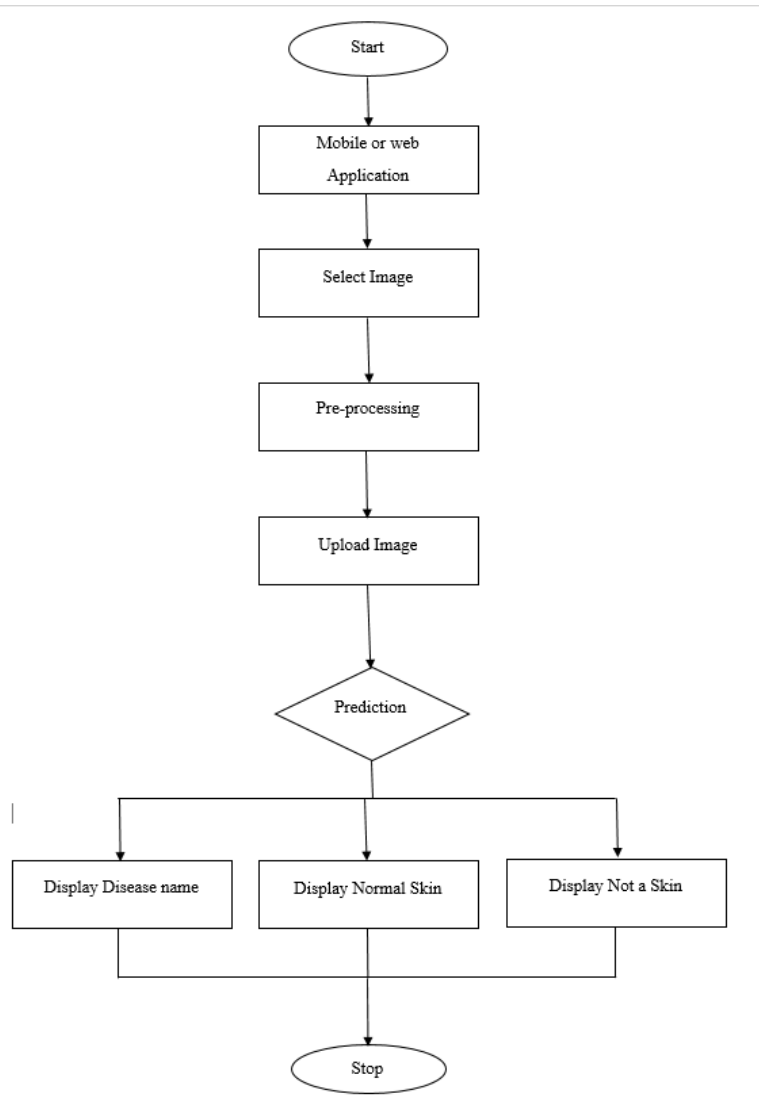

Fig shows the flowchart for skin disease detection using MobileNet Model

Figure shows the flowchart of skin disease identification. Flowchart shows how the system works when input is given considering different scenarios. Initially the image is taken as input through mobile or web application, and the preprocessing of the input image takes place followed by predicting the skin disease if present. If the image does not have any skin disease then display it as normal skin, and not a skin if the image is not considered as a skin.

\section{F. Android Application}

The development of android application that can be useful in identifying the skin diseases using MobileNet Convolutional Neural Networks. The user has to go through few authentication steps in order to access the application. 

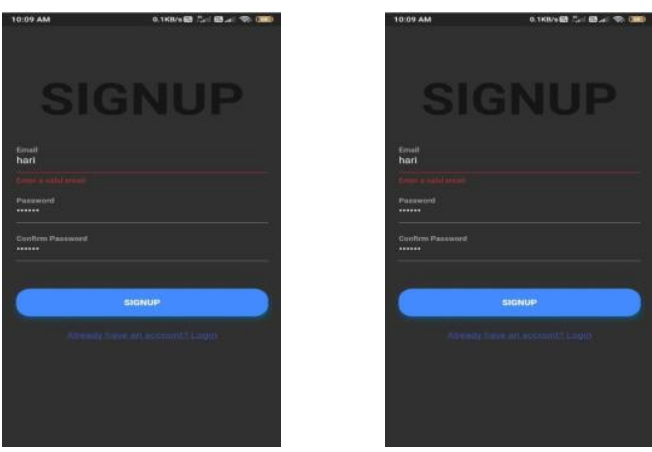

Fig shows the sign up and login page of the application
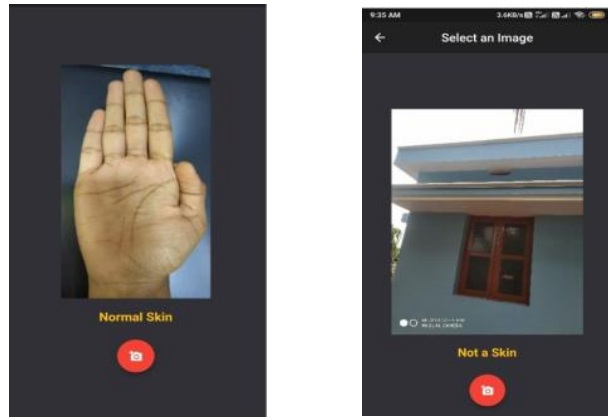

Fig shows the graphical user interface of the app
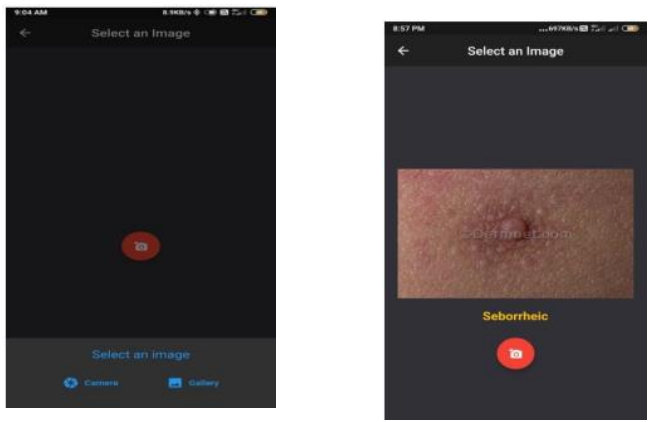

Fig shows the result screen of normal skin and not a skin.

\section{CONCLUSION}

The skin disease detection has become one of the major issue in the medical field and can be solved if it is diagnosed in its early stage. The literature survey mentioned above shows different techniques used in detection of skin diseases across the world. We can see that how application of machine learning helps in lessening the work of dermatologists. User can make use of android app without having extra equipment required which also makes it inexpensive for everyone's reach.

In the proposed approach, which is a android app we have observed that there are vast varieties of disease of the skin found. So it is very difficult to classify all of them. We have taken into the consideration of the dataset which consists of 1000 images each of 6 types of disease namely Acne, Actinic, Eczema, Psoriasis, Seborrhoea, Tinea ringworm. We have used Machine Learning domain and implemented the Convolutional Neural Network Method (CNN) which is used because it is one of the most efficient methodology under machine earning which has delivered about $81 \%$ accuracy.

\section{Future Work}

For future work we are focussing on developing our app more interactive for the users. We can suggest any dermatologist nearby to the user and provide their contact information, so that users can get primary consultation once they are diagnosed by the skin disease. Also the number of diseases detected by our app can also be improved by adding few more diseases. The performance of the model must be precise even if there any issue with the lighting. Finally, quick and accurate prediction must be made to cure the disease at the earlier stage.

\section{REFERENCES}

[1]. Jessica Vellasco, Cherry Pasicon, Jonathan Apuang, "A Smartphone-Based Skin Disease Classification Using Mobilenet Model", International Journal of Advanced Trends in Computer Science and Engineering, Vol 8 2019.

[2]. Ms. Seema Kolkur, Dr. D.R.Kalbande, Dr.Vidya Khakar, "Machine Learning Approches to Multi-Class Human Skin Disease Detection". 
International Journal on Computer Intelligence

Research, vol 14, 2018

[3]. Prem J. Patil, Sagsr J. Buchkule, Varsha S. More, Sanket G. Abhale, "Skin Disease Detecion using Image Processing Technique”. International Research Journal of Engineering and Technolgy (IRJET), vol 7, June 2020.

[4]. Nawal Soliman ALolifi ALEnezi, "A Method of Skin Disease Detection Using Image Processing And Machine Learning”. International Learning and Technology Confeence 2019.

[5]. Shuchi Bhadula, Sachin Sharma, Piyush Juyal, Chitransh Kulshrestha, "Machine Learning Algoritms based Skin Disease Detection". International Journal of Innovative Technology and Exploring Engineering (IJITEE), vol9, Dec 2019.

\section{Cite this article as :}

N. Divya, Deepthi P Dsouza, Hariprasad, "Cureskin Skin Disease Prediction using MobileNet Model", International Journal of Scientific Research in Computer Science, Engineering and Information Technology (IJSRCSEIT), ISSN : 2456-3307, Volume 7 Issue 4, pp. 32-37, July-August 2021. Available at doi : https://doi.org/10.32628/CSEIT21745 Journal URL : https://ijsrcseit.com/CSEIT21745 